\title{
Desarrollo e implementación de un modelo matemático para la evaluación del impacto ambiental de la intrusión marina en el acuífero del río Caplina, Tacna-Perú
}

\author{
E. Rojas Gonzáles, H. Herrera Pamo y Douglas D. Sarango* \\ Departamento de Ingeniería Mecánica de Fluidos, Facultad de Ciencias Físicas, Universidad Nacional \\ Mayor de San Marcos, Apartado Postal 14-0149, Lima, Perú
}

Recibido 03 agosto 2010 - Aceptado 27 setiembre 2010 La sobreexplotación de los acuífero costeros de nuestro país para el abastecimiento de la demanda
de agua para uso domestico y agrícola ha producido en los últimos años un incremento del problema
de la intrusión marina en estos acuíferos. Una forma de diagnosticar y diseñar las soluciones a este
problema, es la modelación matemática en régimen transitorio; que consiste en la solución numérica de
las ecuaciones que gobiernan el flujo de agua en un acuífero, usándose técnicas de diferencias finitas.
La zona de aplicación del modelo desarrollado fue el acuífero del río Caplina en Tacna-Perú. En la
etapa de calibración mensual del modelo, se logró reproducir el comportamiento del acuífero para el
año 2000 con un $95 \%$ de aproximación; es decir con un $5 \%$ de error entre las piezometrías históricas
y las piezometrías obtenidas por el modelo desarrollado. Considerando el futuro incremento del área
agrícola para el período $2000-2004$, se simularon dos caudales de explotación, que dan una nueva
posición de la interfase agua dulce-agua salada. Al simularse con el primer caudal de explotación, los
resultados indican que no representa un peligro para la zona del litoral del valle del Caplina, mientras
que al simularse con el segundo caudal de explotación, los resultados indican que está en peligro la
reserva de agua del acuífero.
Palabras claves: Acuífero Caplina, intrusión marina, modelación numérica, técnicas de diferencias finitas.

\section{Development and implementation of a mathematical model for environmental im- pact of seawater intrusion evaluation in the Capline river aquifer, Tacna, Peru}

The over-exploitation of our country's coastal aquifer to supply water demand for domestic and agricultural use has caused in recent years an increasing problem of seawater intrusion into these aquifers. One way for diagnosis and solutions design to solve this problem came from the mathematical modelling of the transitional regime, which basically consist in the numerical solution of the dynamical equations of water flows in a aquifer by finite difference techniques.

The area of application of the model developed was the Caplina aquifer in Tacna, Peru. The monthly calibration stage, the model achieved reproduce the behaviour of the aquifer for the year 2000 with $95 \%$ of approximation, it means a $5 \%$ error between the historical piezometry and the piezometry obtained for the developed model. Considering the future growth of the agricultural area for the period 2000-2004, two scenarios of exploitation were simulated with the developed model, which give a new position of the interface freshwater-seawater. By simulating the scenario- 1 , the results indicate that not represent a danger to the coastal area of the Caplina valley, while the simulation of the scenario-2, the results indicate that the aquifer's water reserves is endangered.

Keywords: Caplina aquifer, marine intrusion, modeling numeration, finites differences technique.

\footnotetext{
*dsarangoj@unmsm.edu.pe
} 


\section{Introducción}

La sobreexplotación del acuífero de Caplina para el abastecimiento de la demanda de agua de uso agrícola ha producido en los últimos años un incremento de la salinidad del agua extraída, como consecuencia de la presencia de la intrusión marina en el acuífero[1]. Hecho que ha sido comprobado por las evaluaciones que viene efectuando la Dirección General de Aguas y Suelos del INRENA. Muestreos efectuados en el mes de junio del 2000 en pozos cercanos al litoral, indican que la evolución de la concentración salina desde el año 1997 al 2000 a tenido un ascenso, incrementándose entre 3 a 5 veces la concentración inicial.

El sector agricultura desea incrementar la explotación del acuífero mediante la ampliación de la frontera agrícola, que implica la perforación de nuevos pozos de agua subterránea, un primer proyecto con un caudal de explotación de $100 \mathrm{l} / \mathrm{s}$ mediante 05 pozos localizados en el asentamiento-Xl del valle de Caplina, un segundo proyecto igual al primero pero con un caudal incremental de $25 \mathrm{l} / \mathrm{s}$.

Dada la problemática actual de intrusión marina en el acuífero y siendo necesario su análisis y control dentro del acuífero, hemos desarrollado e implementado un modelo matemático para la simulación de la operación del acuífero del Caplina mediante la técnica de diferencias finitas con mallas asimétricas, para ello elaboramos un programa en el lenguaje Visual Basic V.6 para resolver las ecuaciones y para el tratamiento de resultados de utilizamos el modelo Surfer V.8. Con esta metodología hemos simulado la explotación considerando 100 1/s para el primer caso y $125 \mathrm{l} / \mathrm{s}$ para el segundo, para determinar los niveles de intrusión marina en el acuífero del Caplina bajo condiciones de Ghyben-Herzberg.

Los modelos matemáticos permiten la solución de las ecuaciones que rigen el movimiento del agua subterránea en el contexto de las particularidades geométricas, hidrogeológicas, hidrológicas, condiciones de frontera y de explotación que caracterizan al acuífero a modelar[2,3]. Al no existir una solución analítica general, los modelos matemáticos se basan en la aproximación discretizada, que permite formular de forma general el cálculo numérico de la solución de esta ecuación y su expresión se hace particular con la introducción de las restricciones impuestas por la realidad física del acuífero y los criterios del planificador.

En este sentido, al simular situaciones históricas, las respuestas del modelo deben coincidir con los estados previamente conocidos en el acuífero, comprobando hasta que punto el modelo es confiable y es capaz de reproducir el comportamiento real.

\section{Metodología}

La elección de discretizar asimétricamente (poligonalmente) el medio acuífero es por que dentro del área de estudio la información básica no se distribuye uniformemente debido a la posición arbitraria de pozos de explotación dentro del ámbito del acuífero, y se logra resolver el problema de bordes irregulares del contorno acuífero contribuyendo con esto a una reducción del esfuerzo de cómputo.

En base a la discretización asimétrica se desarrollará un modelo matemático que consta de dos partes: la primera que elabora las ecuaciones lineales para cada polígono y la segunda que resuelve el sistema total de ecuaciones.

Para poder obtener el modelo propiamente dicho, se ha seguido el siguiente procedimiento: (i) elección de la discretización del área del acuífero, (ii) método de solución a emplearse, (iii) estructura del modelo, y (iv) calibración ó verificación de parámetros del modelo en régimen transitorio. La fase de utilización del modelo desarrollado es la etapa de simulación, que se realiza con la finalidad de conocer su comportamiento ante una serie de alternativas de explotación factibles, que cumplan restricciones físicas, económicas y técnicas, para esto se tiene que efectuar: (v) simulación de hipótesis de explotación, y (vi) determinación de la interfase agua dulce-agua salada.

\subsection{Discretización del área de estudio}

Se realiza la discretización del medio acuífero en una serie de elementos asimétricos, polígonos. Esta discretización está ligada a la elección del método de cálculo, el tipo de malla que se va a emplear y la dimensión de sus elementos con la finalidad de lograr un ajuste al contorno de área del acuífero.

\subsection{Desarrollo de mallas asimétricas}

La discretización asimétrica del medio acuífero se realiza empleando polígonos de cualquier número de lados, en la figura 1 se muestra un dominio acuífero con una distribución arbitraria de pozos y con márgenes irregulares; subdividida en forma asimétrica por el empleo de polígonos, a cada pozo se le asigna un polígono. 


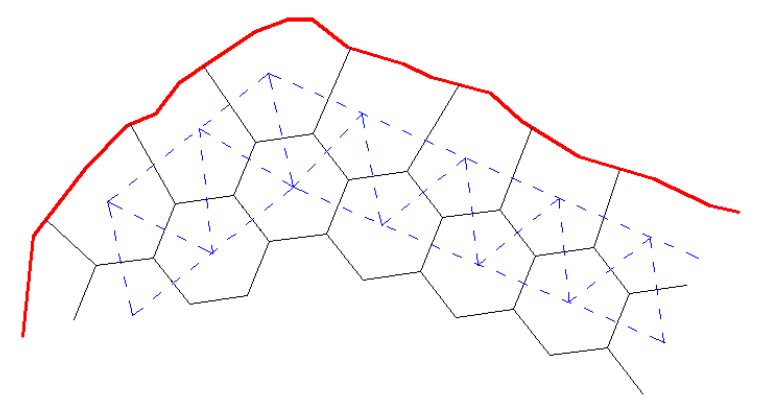

Figura 1: Acuífero con distribución arbitraria de pozos y malla asimétrica.

Cada polígono está definido por el número de caras, la longitud de cara y el espesor del acuífero. Un nodo típico, sus puntos vecinos y el área poligonal se muestra en la figura 2 .

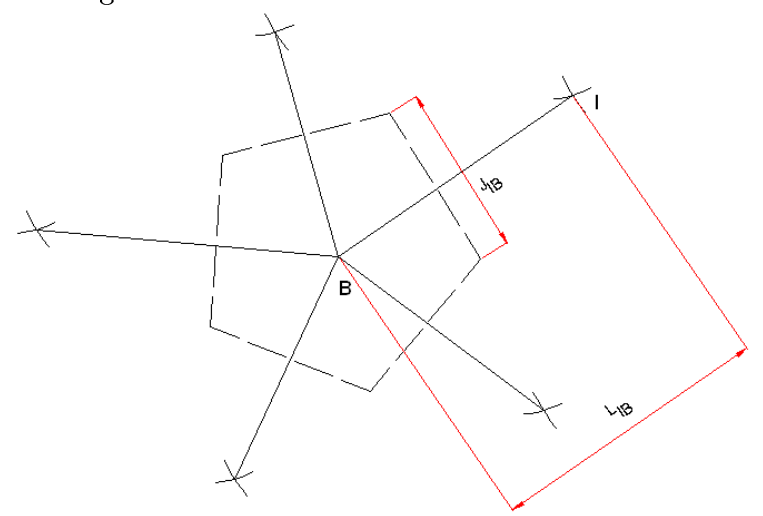

Figura 2: Nodo típico de una malla asimétrica, donde $A_{B}$ es el área de influencia del pozo $B$ y $B_{l}$ son los pozos.

\subsection{Método de la malla poligonal}

Si suponemos la planta del acuífero descompuesta en elementos de forma poligonal, la condición de equilibrio de un prisma elemental que tuviera como base dicho elemento, sería la misma que la estudiada en el apartado anterior, y el balance en un instante de elevación será el volumen entrante por el perímetro igual al volumen extraído más el volumen recargado siendo igual al incremento de volumen almacenado durante el intervalo

$$
d V_{P}+d V_{S}=d V_{T}
$$

Los volúmenes extraídos se consideran negativos y los volúmenes recargados (volumen de inyección) se consideran positivos; evaluando cada uno de estas componentes se tiene el flujo entrante por el perímetro. Asimismo el elemento $B$, para la cara adyacente y en el elemento $l$ se supone una transmisividad constante, de modo que el gradiente, siempre normal a la misma es

$$
\frac{\partial h}{\partial r}=\frac{h_{l}-h_{B}}{L_{l B}}
$$

siendo $L_{l B}$ la distancia entre centros de cada elemento. El volumen entrante total será la suma de los volúmenes entrantes por las distintas caras dadas por

$$
d V_{P}=\sum_{l=1}^{n} \frac{T_{l B} J_{l B}}{L_{l B}}\left(h_{l}-h_{B}\right)
$$

donde $J_{l B}$ es el ancho de la cara, entendiéndose que el índice de sumatoria $l$ se extiende a todos los nudos $l$ que rodean al elemento $B, T_{l B}$ es la trasmisividad de la cara común entre los nodos $l$ y $B, h_{l}$ y $h_{B}$ son los niveles piezométricos de los polígonos $l$ y $B$.

El volumen extraído y el volumen recargado es un dato para cada prisma de modo que

$$
d V_{S}=Q_{B} \Delta t
$$

donde $Q_{B}$ es la velocidad de flujo volumétrico por unidad de área en el nodo $B$ (recarga o bombeo).

La variación de almacenamiento, suponiendo que $h$ es constante en todo el polígono, así como su coeficiente de almacenamiento $S$ tendremos

$$
d V_{S}=\left(h_{B}-h_{B}^{\prime}\right) S_{B} A_{B}
$$

donde $h_{B}^{\prime}$ el valor del nivel en el polígono en el instante anterior al cálculo, $A_{B}$ es el área del elemento y $S_{B}$ es el coeficiente del almacenamiento medio.

De acuerdo con lo anterior la relación de equilibrio de un elemento volumétrico finito, denominada conductancia del ramal $l B$ será expresado como

$$
Y_{l B}=\frac{J_{l B} K_{l B}}{L_{l B}},
$$

donde $J_{l B}$ es la longitud de la cara común a los dos polígonos $l$ y $B, K_{l B}$ es el coeficiente de permeabilidad en el punto medio de los nodos $l$ y $B$.

El resultado de discretizar el espacio acuífero utilizando una malla asimétrica está dado por la ecuación de Mac Neal,

$$
\sum_{l=1}^{n}\left(h_{l}-h_{B}\right) Y_{l B}=A_{B} S_{B} \frac{\partial h_{B}}{\partial t} A_{B} Q_{B}
$$

donde la conductancia se reescribe como

$$
Y_{l B}=\frac{J_{l B} T_{l B}}{L_{l B} b_{B}}
$$

observando que $b_{B}$ es el espesor del acuífero en el nodo $B$.

El lado izquierdo de la Ec.7 es la suma de los flujos del subsuelo entre un área dada y sus áreas adyacentes. La velocidad del cambio de almacenamiento está dada por el primer término del lado derecho, y el segundo término representa la velocidad de flujo superficial de 
la superficie del suelo hacia adentro ó fuera de la zona de saturación del área unitaria dada.

Discretizando la derivada de tiempo en la Ec.7 por diferencias finitas hacia atrás (implícitas), se tiene[4]

$$
\sum_{l=1}^{n}\left(h_{l}^{J}-h_{B}^{J}\right) Y_{l B}=\frac{A_{B} S_{B}}{\Delta t}\left(h_{B}^{J}-h_{B}^{J-1}\right)+A_{B} Q_{B}
$$

donde el índice superior $J$ denota el tiempo, y el subíndice $l$ denota el conjunto de nodos adyacentes al nodo de interés o sea, el nodo B.

El método de solución empleado es el implícito, el proceso se dice totalmente implícito si el potencial de un punto al instante $t+\Delta t$ depende de los potenciales de los puntos periféricos al instante $t+\Delta t$. Para cada período se resuelve un sistema de ecuaciones simultáneas, siendo el número de ecuaciones igual al número de pozos o polígonos considerados. La estructura de la matriz de coeficientes tiene la diagonal dominante, aspecto que resulta muy importante para lograr la aplicación de técnicas altamente eficientes para la solución de ecuaciones simultáneas, utilizándose el algoritmo de Gauss-Seidel, cuyas características de solución es eficiente para este tipo de problemas.

De otro lado, la ubicación de la interfase agua dulce-agua salada, que representa el problema de intrusión marina, se determina aplicando la condición de Ghyben-Herzberg, que se expresa por la relación siguiente

$$
h_{s}=40 h_{f}
$$

donde $h_{S}$ es la profundidad a la cual se halla la interfase agua dulce-agua salada, $h_{f}$ es el nivel del agua subterránea y 40 es el valor deducido de las densidades de agua dulce y agua salada (Ghyben-Herzberg).

\section{Información utilizada}

El acuífero del valle Caplina, está ubicado en el departamento de Tacna, entre $18^{\circ} 20^{\prime}$ de latitud sur y $70^{\circ} 10^{\prime}$ de longitud oeste, la zona específica comprende en general el cono deyectivo del río Caplina, sobre el cual se sitúan las Pampas de la Yarada y Hospicio, considerándose como límite del estudio la extensión entre la ciudad de Tacna, el litoral y los afloramientos rocosos que rodean el cono aluvial.

La información correspondiente a datos de piezometría histórica utilizada es para el acuífero superficial, básicamente la del año 2000, que sirvió como base para la etapa de calibración del modelo por ser la más consistente. Los valores de transmisividad son de $1 \times 10^{-2}$ a $5 \times 10^{-2}$ y los coeficientes de almacenamiento están en el rango de $0.3 \%$ a $6 \%$.

El primer caudal de explotación a ser simulado en el período 2000-2004, comprende $100 \mathrm{l}$ /s de sobreexplotación, con respecto a la explotación del año 2000, y corresponde al Asentamiento-X a operar en enero del 2002, mientras que el segundo caudal de explotación consiste en explotar un caudal de $125 \mathrm{l} / \mathrm{s}$, a implementarse en el Asentamiento-Y que entró en operación en enero del 2003.

\section{Desarrollo e implementación del mo- delo matemático del acuífero de $\mathrm{Ca}$ - plina}

La implementación y el desarrollo del modelo constan de la siguientes partes:

\subsection{Etapa de desarrollo e implementación del modelo}

En esta etapa se desarrolló un programa en lenguaje Visual Basic para la implementación del modelo matemático de explotación de aguas subterránea del acuífero del Caplina, y luego se desarrolló una interfase con el programa Surfer para el tratamiento gráfico de los resultados del modelo[2,3,5-9].

\subsection{Etapa de calibración}

En esta etapa se busca reproducir las piezometrías observadas históricamente, considerados como estado final del acuífero. El procedimiento seguido es el siguiente: a) recopilación y análisis de la información, b) selección del periodo de calibración, c) límites del acuífero, d) discretización espacial del acuífero, e) configuración de la malla, f) determinación de las propiedades hidrogeológicas, g) flujos impuestos, h) estado inicial del acuífero, i) condiciones de frontera[2,3].

\subsection{Etapa de simulación}

Una vez obtenida la confiabilidad del modelo en la etapa de calibración, este se utilizó para obtener los estados finales del acuífero sometido a los regímenes de explotación siguiente: (a) hipótesis de explotación N. ${ }^{\circ}$ 1: el acuífero es sometido a un caudal de explotación de $100 \mathrm{l} / \mathrm{s}$ (05 pozos), y (b) hipótesis de explotación N. ${ }^{\circ}$ 2: el caudal de explotación del acuífero es de $125 \mathrm{l} / \mathrm{s}$ (06 pozos).

\section{Resultados y discusión}

Los resultados de la simulación numérica están discutidos considerando las etapas siguientes: 


\subsection{De la etapa de desarrollo e implemen- tación del modelo}

Se implementó un programa en lenguaje Visual Basic 6.0 para la solución de la Ec.9 para el acuífero del Caplina, con almacenamiento de los resultados en hojas de cálculo del Excel, para ser usados en el modelo Surfer V.8, para la visualización y analisis de los resultados en forma gráfica.

El programa consta de 6 formularios, el primero se refiere al ingreso al programa mismo y el segundo al cuarto formulario que visualiza las tablas de entrada de datos previamente llenados en una base de datos. El quinto formulario procede a realizar el cálculo de la piezometría del mes de estudio, es decir el proceso de calibración y comprobando la aproximación de los valores verdaderos con un error menor del $5 \%$. Para finalizar con el último formulario que consta del cálculo anual de las piezometrías de los polígonos de cada uno de los meses, utilizandose el método de Gauss-Seidel.

Se elaboró el Manual de Usuario del modelo desarrollado, el cual permite una compresión rápida de la entrada de datos, procesos dentro del modelo, modo de almacenamiento de los datos y los resultados, así como la presentación de los mismos.

\subsection{De la discretización del área de estudio}

Se discretizó el área de estudio del acuífero del Caplina, obteniéndose 40 polígonos, de los cuales 34 son polígonos de cuatro lados y 6 son de más lados, ver Figura 3. De este plano se han obtenido características de cada polígono como su número de caras, longitud de cada cara, longitud entre centroides de polígonos adyacentes, área del sector acuífero inscrito en el polígono, etc.

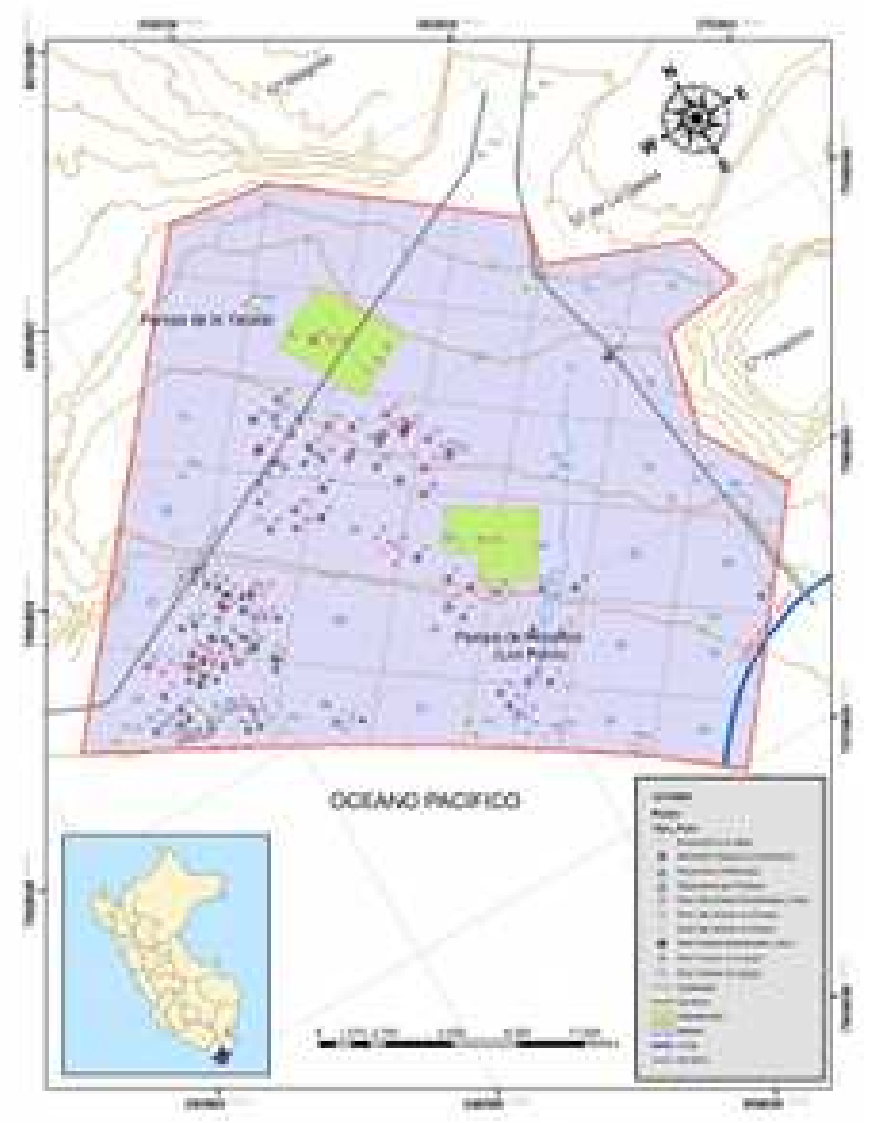

Figura 3: Ubicación y discretización de la zona de estudio. Acuífero del Caplina. 


\subsection{De la etapa de calibración}

La etapa de calibración se divide en dos fases, la primera en la que se obtiene los datos de entrada al modelo y la segunda donde se ajustan o verifican estos datos. Los datos necesarios de entrada al modelo son las características individuales de cada uno de los elementos de la discretización y el área acuífera que inscriben, los cuales serán a nivel mensual para el período de calibración, año 2000, información que se encuentra en la base de datos del modelo.
En la Figura 4, se muestran los resultados de las etapas de calibración seguidas para período del mes de enero-1999 a diciembre 2000, observándose el valor de piezometría calculada por el modelo y la piezometría histórica registrada en el área de estudio.

De la comparación de la piezometría calculada por el modelo y la histórica se aprecia el grado de calibración obtenida, que fue menor o igual al $5 \%$. Analizando los resultados se obtiene una diferencia máxima de $0.05 \mathrm{~m}$. en los polígonos 34 y 36 con lo cual se logró representar el sistema real con $3 \%$ de error.
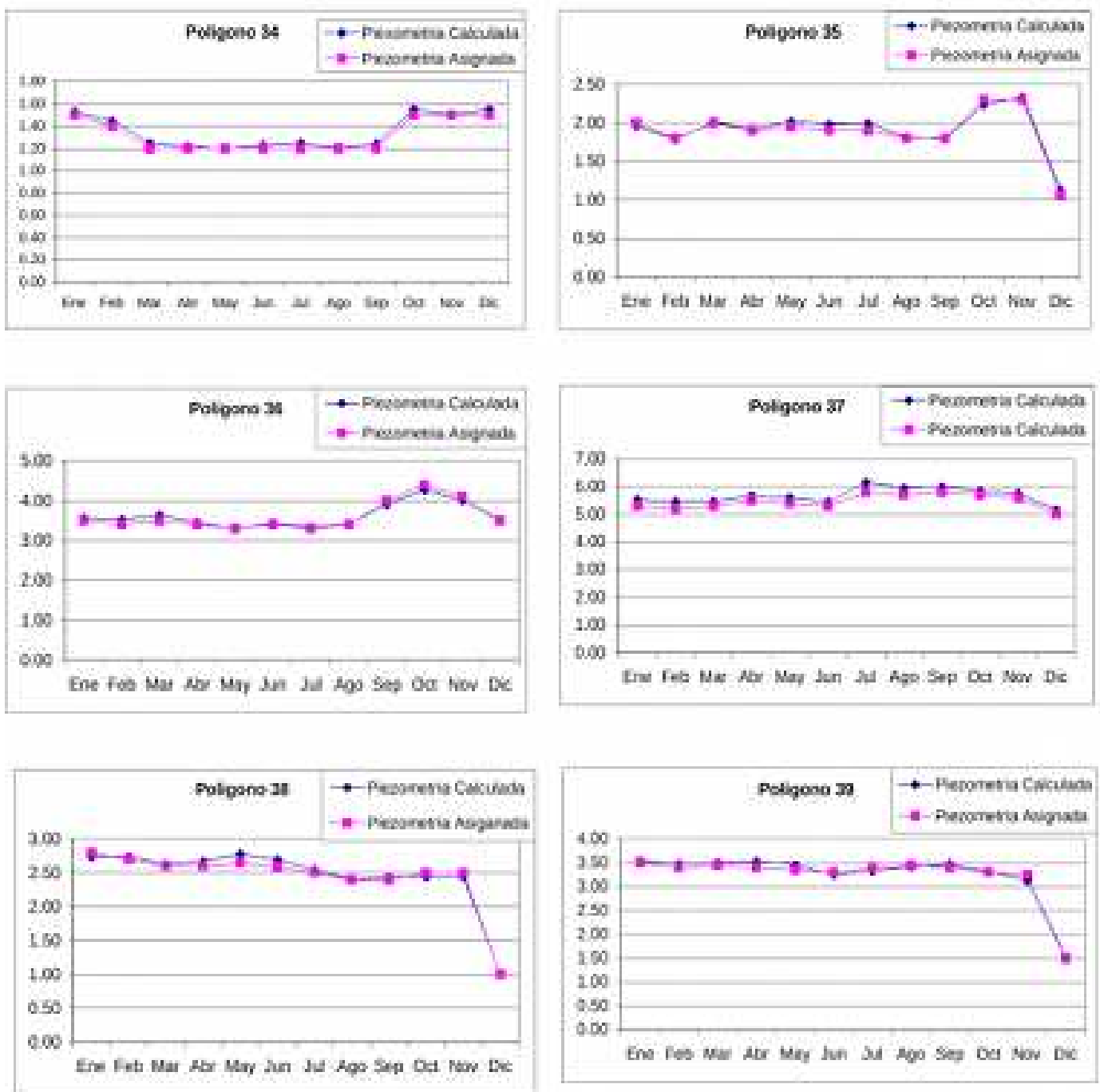

Figura 4: Resultados de la etapa de calibración a nivel mensual correspondiente al año 2000. 


\subsection{Resultados de la Etapa de Simulación}

La simulación de las hipótesis de explotación, se realizaron en régimen transitorio utilizando el modelo desarrollado e implementado en la etapa de calibración, esto con la finalidad de conocer el riesgo de deprimir la napa hasta niveles peligrosos que conlleve a incrementar el fenómeno de intrusión marina en la parte litoral del valle Caplina en el período 2001-2004.

La hipótesis de explotación-1 consiste en incrementar la explotación actual del año 2000, en $100 \mathrm{l}$ /s que son los requerimientos del Asentamiento-X, a implementarse en enero 2002, que ha sido distribuido en las mallas N. ${ }^{\circ} 8$ y 9 . Calculándose la piezometría de las mallas que conforman la discretización del área de estudio, período 2001-2004.

Para determinar la posición de la interfase agua dulce-agua salada se ha tomado como referencia el descenso piezométrico con relación a la piezometría de diciembre del 2000 en las mallas de las zona litoral del
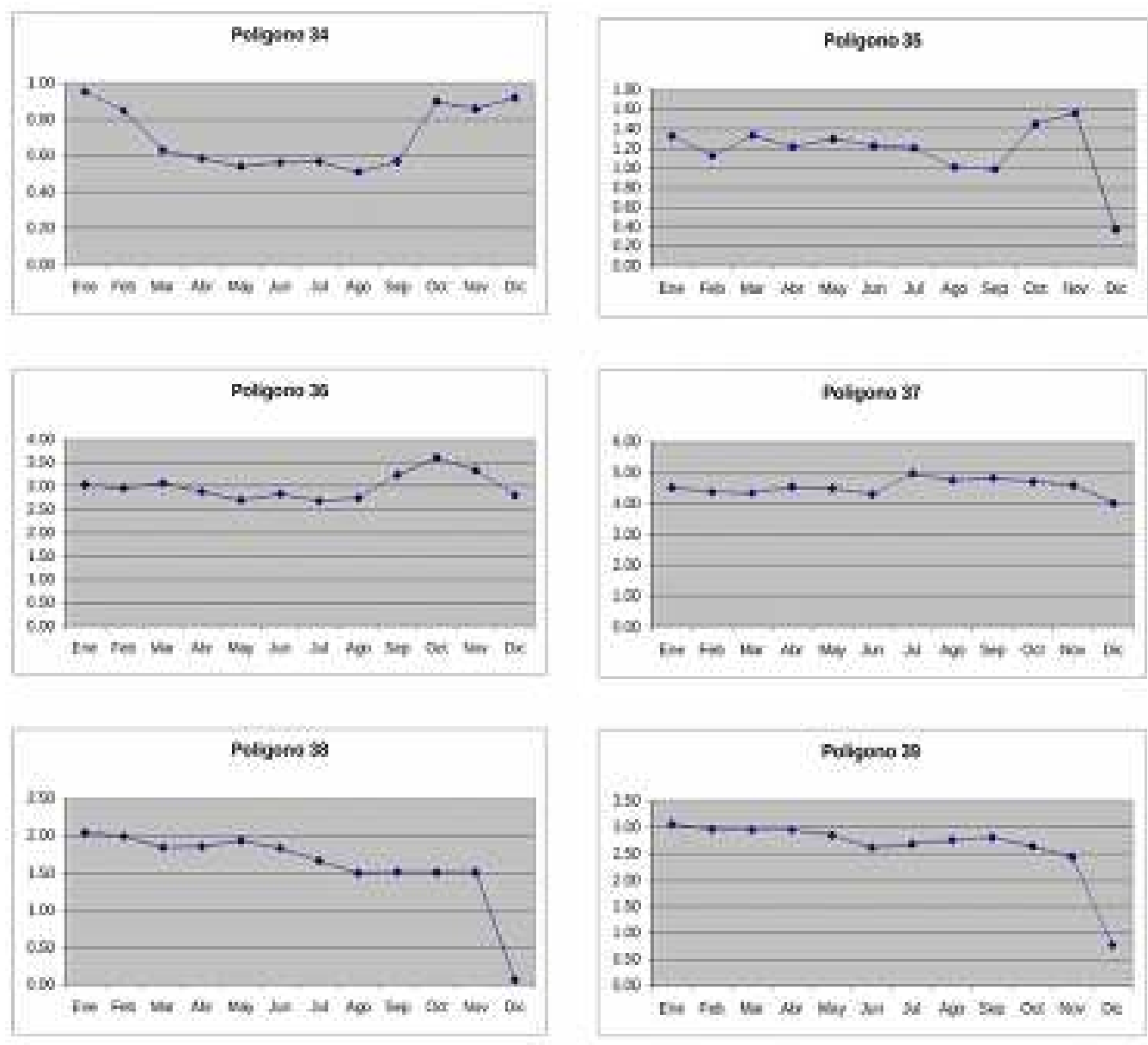

Figura 5: Piezometría calculada, la etapa de simulación corresponde al año 2004 con el caudal de explotaciónde 125 l/s.

valle Caplina (ver figura 3). El rango de variación de los abatimientos puntuales ocurridos en la zona litoral, para los polígonos 34 al 39 ubicados en la zona litoral son como mínimo de $0.64 \mathrm{~m}$. y $0.89 \mathrm{~m}$. como máximo, ocurriendo estos valores en los polígonos 36 y 37.

La hipótesis de explotación-2, consiste en estudiar el comportamiento del acuífero sometido a una explotación adicional de $125 \mathrm{l} / \mathrm{s}$, respecto a la explotación existente para 2000. Este incremento de caudal incluye el correspondiente a la hipótesis-1, $100 \mathrm{l} / \mathrm{s}$, y el caudal restante de $25 \mathrm{l} / \mathrm{s}$, corresponde al incremento de explotación en el Asentamiento-Y, el cual será para enero 2003, y se distribuyó en la malla N. ${ }^{\circ} 23$ y 24 .

La Figura 5 muestra los diagramas piezométricos para las mallas de la zona litoral del valle Caplina, determinándose el rango de variación de los abatimientos puntuales para el período 2000-2004, ocurridos en la zona litoral, obteniéndose como mínimo de $0.23 \mathrm{~m}$. y como máximo $5.1 \mathrm{~m}$., ocurriendo estos valores en los polígonos 39 y 38 .
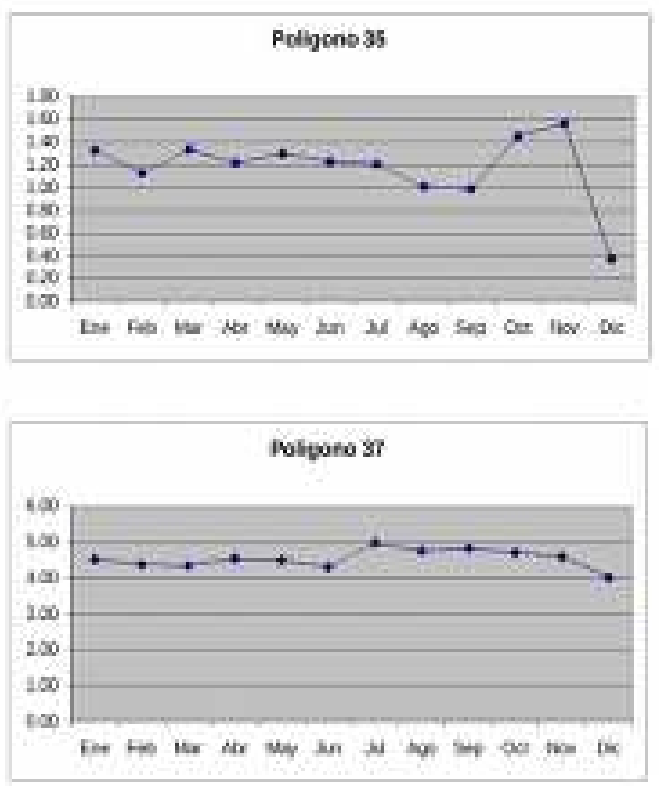


\subsection{De la intrusión marina}

Teniendo en cuenta la piezometría calculada por el modelo para cada hipótesis de explotación simulada $h_{f}^{\prime}$, y considerando la Ec.10 se obtendrá la nueva posición adoptada por la interfase para el período simulado, 2001-2004.

En el Cuadro 1, se presenta las variaciones puntuales de la interfase marina para la zona litoral del valle Caplina considerando la hipótesis de explotación-1, observándose que, las variaciones son aceptables para las mallas que limitan con el litoral, mallas N. ${ }^{\circ} 34,35,36$, 37,38 y 39 , por ser valores que no son peligrosos para la contaminación del acuífero, esto en relación a la posición puntual de la interfase marina con relación al nivel medio del mar.

\begin{tabular}{lccl}
\hline Polígono & $\begin{array}{c}\text { Interfase } \\
\text { Dic 2000 }\end{array}$ & $\begin{array}{c}\text { Interfase } \\
\text { Dic 2004 H1 }\end{array}$ & Variación \\
\hline 34 & 62.10 & 32.80 & 29.21 \\
35 & 45.10 & 13.60 & 31.50 \\
36 & 139.15 & 113.60 & 25.55 \\
37 & 206.70 & 171.20 & 35.50 \\
38 & 40.12 & 11.20 & 28.92 \\
39 & 58.87 & 33.20 & 25.67 \\
\hline
\end{tabular}

Tabla 1: Variación puntual de la interfase marina, período 2000-2004: hipótesis de explotación-1.

\begin{tabular}{lccl}
\hline Polígono & $\begin{array}{c}\text { Interfase } \\
\text { Dic 2000 }\end{array}$ & $\begin{array}{c}\text { Interfase } \\
\text { Dic 2004 H1 H2 }\end{array}$ & Diferencia \\
\hline 34 & 62.10 & 36.77 & 24.24 \\
35 & 45.10 & 15.06 & 30.04 \\
36 & 139.15 & 112.44 & 26.71 \\
37 & 206.70 & 160.58 & 46.12 \\
38 & 40.12 & 2.74 & 37.37 \\
39 & 58.87 & 31.04 & 27.83 \\
\hline
\end{tabular}

Tabla 2: Variación puntual de la interfase marina, período 2000-2004: hipótesis de explotación-2.

En lo que respecta a las variaciones puntuales ocasionados al entrar en ejecución la hipótesis de explotación-2, se consideran demasiadas peligrosas, por estar muy cerca del nivel medio del mar, por considerarse que la posición de la interfase delimita una zona más o menos grande donde ocurren fenómenos de dispersión y difusión por lo cual la interfase estaría introduciéndose en el dominio del acuífero del Caplina, esto como consecuencia de un desequilibrio entre la explotación y la alimentación del acuífero, ver Cuadro 2.

En la Figuras 6, se presenta gráficamente la posición de la interfase marina para el valle del Caplina, en la zona litoral, al simularse los caudales de explotación de 100 l/s para el período 2000 al 2004. Para el caudal de explotación de $125 \mathrm{l} / \mathrm{s}$ no está siendo mostrado el gráfico de intrusión porque a las escalas presentadas son muy similares a la de $100 \mathrm{l} / \mathrm{s}$.

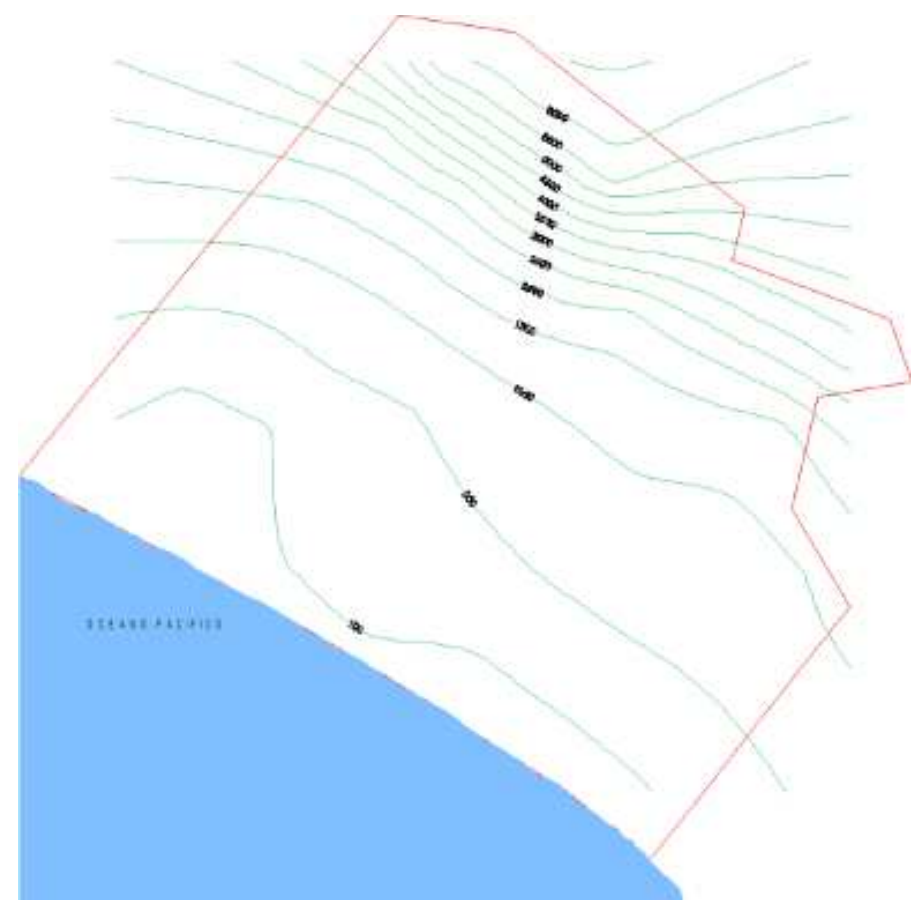

Figura 6: Posición de la interfase agua dulce-agua salada con el caudal de explotación de 100 1/s. 


\section{Conclusiones y Recomendaciones}

El presente trabajo nos permite obtener varias conclusiones y también nos permite establecer una serie de recomendaciones, veamos.

\subsection{Conclusiones}

Se desarrolló e implementó un modelo matemático para el análisis y la evaluación del impacto ambiental de la intrusión marina para el acuífero del valle Caplina, en Tacna, Perú, utilizándose una discretización asimétrica y usando la técnica de diferencias fínitas.

El modelo elaborado fue desarrollado y programado en VISUAL BASIC V.6, que almacena los resultados en Excel, para luego ser utilizados por el modelo SURFER V.8 para su tratamiento gráfico.

Asimismo se ha elaborado un Manual de Usuario, que permite un manejo fácil del modelo por el usuario.

En la etapa de calibración mensual del modelo, se logró reproducir el comportamiento del acuífero para el año 2000 con un $95 \%$ de aproximación; es decir con un $5 \%$ de error entre las piezometrías históricas y las obtenidas por el modelo.

La hipótesis de explotación 1 simulada para el período 2000-2004, que comprende $100 \mathrm{l} / \mathrm{s}$ de sobreexplotación, con respecto a la explotación del año 2000, da como resultados una variación de los abatimientos puntuales, siendo el mínimo de $0.64 \mathrm{~m}$. y $0.89 \mathrm{~m}$. como el máximo, en los polígonos 36 y 37 de la zona litoral del valle del Caplina. Mientras que la simulación de la hipótesis de explotación 2, da como abatimiento un mínimo de $0.23 \mathrm{~m}$. y un máximo de $5.1 \mathrm{~m}$., localizados en los polígonos 39 y 38 de la zona litoral del valle.

Con respecto a la posición de la interfase agua dulceagua salada, de los resultados obtenidos al simularse las hipótesis de explotación 1 y 2 , se determinó que se debe implementar la hipótesis de explotación 1 por no representar un peligro para la zona litoral del valle del Caplina.

\subsection{Recomendaciones}

Se recomienda utilizar en modelo desarrollado e implementado para la evaluación del impacto ambiental de la intrusión marina en el acuífero del río Caplina, Tacna, Perú.

Se recomienda ejecutar la hipótesis 1 bajo una adecuada política de explotación de la zona litoral del valle Caplina, tratando de no ocasionar una reducción significativa en la capacidad de captación de los pozos ya existentes en esta zona.

Se recomienda no incrementar la explotación en la zona litoral comprendida entre las mallas N. ${ }^{\circ} 34$ a la N. ${ }^{\circ} 40$, para no provocar descensos considerables del potencial piezométrico de agua dulce, que se sumaría a los provocados por la ejecución de la hipótesis recomendada.

\section{Agradecimientos}

El presente trabajo fue realizado con el apoyo del Vicerrectorado Académico de la UNMSM a través del Programa de Iniciación Científica. J.R.G y H.P.H. agradecen el apoyo recibido.

\section{Referencias}

[1] E. Custodio y M.R. Lamas, Hidrologia Subterránea, tomo II, Editorial Omega S.A., Barcelona (1983).

[2] R. Amisial y H. Jegat, Aprovechamiento y modelos de aguas subterráneas, CIDIAT, Mérida (1976).

[3] J. Bear y A. Verruijt, Modelling Groundwater Flow and Pollution, D. Reidel Publishing Company, Dordrecht (1987).

[4] Warren A. Hall y John A. Dracup, Ingeniería de sistema en recursos hidráulicos, C.E.C.S.A., México (1984).
[5] A. Nieves y F. Domínguez, Métodos númericos aplicados a la Ingeneiría, C.E.C.S.A., México (1998).

[6] Golden Software Inc., Surfer for Windows User's Guide (1994).

[7] M.García M., Lenguaje de Programación Fortran 95, Editorial Paraninfo, Madrid (1990).

[8] J.D. Sarango, Análisis del problema de intrusión marina del acuífero de Chilca mediante modelamiento númerico, Rev. Inv. Fis. 9, 29 (2006).

[9] A. Verruijt, Theory of Groundwater Flow, Delft University of Thechnology, Amsterdam (1970). 